\title{
MANEJO LAPAROSCÓPICO DE QUISTES OVÁRICOS COMPLICADOS O PERSISTENTES DURANTE EL EMBARAZO
}

\author{
José Luis Troncoso J. ${ }^{1}$, Paolo Ricci A. ${ }^{1}$, Jaime Albornoz V. ${ }^{2}$, Antonio Mackenna I. ${ }^{2}$ \\ ${ }^{1}$ Unidad de Ginecología, ${ }^{2}$ Unidad de Medicina Reproductiva, Departamento de Obstetricia y Ginecología, Clínica Las \\ Condes.
}

\section{RESUMEN}

Antecedentes: El incremento en el hallazgo de quistes anexiales durante el embarazo sería el mayor uso de técnicas de reproducción asistida con hiperestimulación ovárica controlada. La complicación más frecuente sigue siendo la torsión anexial. Objetivo: Revisar el manejo de quistes anexiales complicados o persistentes, durante el embarazo, con resolución laparoscópica. Método: Revisión retrospectiva de 9 pacientes consecutivas, ingresadas al Departamento de Obstetricia y Ginecología Clínica Las Condes. Resultados: La edad gestacional de ingreso fue entre $5+5$ y 27 semanas. Cinco casos fueron producto de fertilización asistida y cuatro espontáneos. Ocho $(85,6 \%)$ embarazadas, ingresaron por dolor abdominal agudo. Otro caso ingresó a cirugía electiva por quiste ovárico persistente. Los quistes tenían entre 6 y $13 \mathrm{~cm}$. En 5 se realizó sólo destorsión ovárica, en 1 ooforectomía y en 1 quistectomía. Se realizó punción, destorsión y fijación a ligamento útero ovárico, bilateral, en la otra paciente. Se registró un caso de fiebre en el postoperatorio. El alta hospitalaria fue a las 48 horas en $6(67 \%)$, a las 72 en 2 y a las 96 en 1. El estudio biópsico diferido en 3 pacientes demostró: 1 quiste seroso paratubario, 1 quiste luteínico parcialmente hemorrágico y 1 infarto anexial. Todos los embarazos evolucionaron normalmente. Conclusión: Nuestra experiencia confirma que en el embarazo la laparoscopía es una buena opción al requerir una cirugía por quiste anexial complicado o persistente.

\section{PALABRAS CLAVE: Torsión anexial, laparoscopia, hiperestimulación ovárica, embarazo}

\section{SUMMARY}

Background: Recent reviews show an increase in the finding of adnexal cysts during pregnancy. One reason could be the use of assisted reproductive techniques with controlled ovarian hyperstimulation. Objective: To review the experience in managing via laparoscopy complicated or persistent adnexal cysts during pregnancy. Method: Retrospective review of 9 consecutive patients, admitted at the Department of Obstetrics and Gynecology, Clinica Las Condes. Results: The gestational age was between $5+5$ and 27 weeks. Five were the product of assisted fertilization and four spontaneous. Eight (85.6\%) pregnant women were admitted by emergency room because of acute abdominal pain and another one went through elective surgery for persistent ovarian cyst. Preoperative diagnosis of the 8 patients admitted by emergency: adnexal cyst complicated with torsion in 6 ( 1 adnexal infarction) and 2 cases of acute abdomen. The cysts were between 6 and $13 \mathrm{~cm}$. Type of surgery: in 5, only detorsion; ovarian oophorectomy in 1 and cystectomy in one. Puncture, detorsion and bilateral fixation of uterine ovarian ligament, in another patient. One case required conversion to laparotomy with adnexectomy. Postoperative fever was observed in one patient. Discharge was at $48 \mathrm{hrs}$ 
in $6(67 \%)$ cases, at $72 \mathrm{hrs}$. in 2 and $96 \mathrm{hrs}$. in 1. Deferred biopsy performed in 3 patients showed: paratubarian serous cyst, partially hemorrhagic luteal cyst, adnexal infarction. All pregnancies went on normally. Premature births are not reported. Conclusion: Our experience suggests that laparoscopic approach is a valid option for complicated or persistent adnexal cysts in pregnant women.

\section{KEY WORDS: Adnexal torsion, laparoscopy, ovarian hiperestimulation, pregnancy}

\section{INTRODUCCIÓN}

El hallazgo de una lesión quística anexial, simple o compleja, en el embarazo, no es un hecho infrecuente. Estudios recientes nos muestran cifras de hasta un 4\%, mientras que las revisiones clásicas reportaban una incidencia de 1 en 2.300 embarazos (1). Con el advenimiento de las técnicas de reproducción asistida e hiperestimulación ovárica controlada, se agrega un factor de riesgo adicional (2). Por otro lado, la estimulación ovárica y el síndrome de hiperestimulación ovárico (SHO) se asocian a un incremento en el riesgo de torsión anexial, por la presencia de ovarios quísticos aumentados de volumen (2-4). La incidencia de torsión anexial después de fertilización in vitro (FIV), varía entre $0,08 \%$ y $0,13 \%$, según distintos estudios (5-7). Sin embargo, en mujeres con torsión anexial, la asociación con SHO fluctúa entre 1\% - 33\% $(4,8,9)$. Al lograrse un embarazo con hiperestimulación ovárica y más aún en la presencia de SHO, los quistes ováricos persisten y esto prolonga el período de riesgo de torsión anexial (10).

Los quistes anexiales presentes en un embarazo espontáneo, requieren cirugía en aproximadamente 1 en 1.300 recién nacidos vivos (11-13). La mayoría de estos quistes anexiales son benignos: quistes dermoides y cistoadenomas serosos $(14,15)$. Sin embargo, un quiste anexial complejo persistente, siempre plantea la posibilidad de una neoplasia maligna con cifras entre 2,3 y $5 \%(14,16$ 19).

Las complicaciones asociadas a quistes anexiales y embarazo incluyen cirugía de urgencia por dolor, torsión y hemorragia, constituyendo emergencias que se pueden desencadenar, también, durante el parto o puerperio.

La torsión es la complicación más frecuente de los quistes anexiales, afectando hasta un $25 \%$ de los casos (3). Por esta razón, uno de los tópicos de permanente discusión, es la indicación quirúrgica de una lesión quística anexial simple, persistente y asintomática, durante el embarazo. Algunos autores recomiendan considerar la cirugía para evitar las complicaciones ya descritas, mientras que otros son partidarios de mantener una estricta observación y control, para evitar las potenciales desventajas de una cirugía durante embarazo $(11,16)$. Por estas razones, algunos prefieren postergar una eventual intervención en caso de ser necesaria, hasta el segundo trimestre.

Previo al advenimiento de las técnicas laparoscópicas, estas pacientes eran abordadas quirúrgicamente por vía laparotómica, con todos los riesgos y complicaciones inherentes a una cirugía abierta asociada a un útero grávido. Sin embargo, en sus inicios, la aproximación laparoscópica estuvo vedada por muchos años, considerándose el embarazo una contraindicación absoluta al desconocerse los efectos de un aumento de la presión intraabdominal, sobre el útero gestante y los efectos del neumoperitoneo con $\mathrm{CO} 2$ sobre el feto. Este concepto cambió radicalmente en la última década, debido a que distintos estudios demostraron que no había diferencia en la evolución y bienestar fetal al comparar laparotomía y laparoscopia durante el embarazo (20). Actualmente son pocos los casos que se reportan de conversión a cirugía abierta, llegando a no más de un $3 \%$.

Las ventajas de la cirugía laparoscópica en el embarazo incluyen menor manipulación uterina, reducción del tiempo operatorio, deambulación y realimentación precoz, disminución de eventos tromboembólicos y alta precoz. Es interesante considerar que al evitarse una cicatriz abdominal con un abdomen en crecimiento, también hay un tema estético y de confort para la paciente.

El presente trabajo tiene como objetivo revisar la experiencia en el manejo de quistes anexiales complicados o persistentes, durante el embarazo, con resolución por vía laparoscópica.

\section{PACIENTES Y MÉTODO}

Revisión retrospectiva de 9 embarazadas, ingresadas por quistes anexiales complicados o persistentes, resueltos por medio de laparoscopia. Ocho casos ingresaron como urgencia y 1 por quiste anexial persistente. El protocolo de revisión incluyó los siguientes parámetros: tipo de embarazo, sus características, edad gestacional, motivo de consulta, diagnóstico clínico y ecográfico pre-operatorio, tipo de abordaje laparoscópico, técnica quirúrgica, tiempo operatorio, días de hospitalización, complicaciones, anatomía patológica y resolución del embarazo. Se revisaron las fichas clínicas de 
cada caso y en aquellos que existían se revisó el video de la cirugía.

\section{RESULTADOS}

La edad de las 9 pacientes fluctuó entre 23 y 36 años, con una media de 31 años. La edad gestacional de ingreso fue entre $5+5$ y 27 semanas (Tabla I).

De los nueve embarazos, 5 fueron producto de fertilización asistida y cuatro espontáneos. Los casos de fertilización asistida se desglosan en: 2 FIV y 3 por inseminación intrauterina (IIU). Todos estos casos presentaron algún grado de síndrome de hiperestimulación ovárica. Hubo 2 casos de embarazos gemelares; 1 doble (bicorial, biamniótico) y 1 embarazo triple (bicorial-triamniótico) (Tabla I).

En 8 casos se realizó cirugía de urgencia y en el restante, el procedimiento fue electivo, por persistencia de un quiste anexial que fue un hallazgo durante las ecografías de rutina. En las $8(85,6 \%)$ embarazadas ingresadas por urgencia, el motivo fue dolor abdominal agudo. Presentándose como dolor persistente en hipogastrio y/o fosas ilíacas en 7 casos y como dolor en flanco izquierdo en otra.

Al ingreso se planteó como hipótesis diagnóstica, cólico nefrítico en 3 casos. Una vez que se completó la evaluación con exámenes, se indicó una cirugía de urgencia en las 8 pacientes, bajo los diagnósticos pre-operatorios de: quiste anexial complicado con torsión en 6 casos ( 1 con infarto anexial) y 2 casos de abdomen agudo (Tabla II).

Con respecto al único caso de cirugía electiva, realizado a las 19 semanas de embarazo, el diagnóstico pre-operatorio fue de lesión quística anexial derecha persistente.
La visión directa durante la entrada primaria, fue realizada con cámara a través de trocar de 10 $\mathrm{mm}$ en 2 casos. En los otros se realizó una entrada ciega. En 8 de los casos se optó por la punción umbilical clásica y sólo en 1 caso fue supraumbilical. La presión de CO2 fluctuó entre 15 y $20 \mathrm{mmHg}$, con flujos entre 2 y 3 litros por minuto.

Los tiempos operatorios estuvieron entre 25 y 60 minutos, con un promedio de 44 minutos. Los diámetros de los quistes fueron entre 6 y $13 \mathrm{~cm}$, con un promedio de $8,6 \mathrm{~cm}$. En 5 casos se realizó sólo destorsión ovárica, en 1 ooforectomía y quistectomía en otro. Se realizó punción, destorsión y fijación a ligamento útero ovárico, bilateral, en la paciente restante (Tabla II). Fue necesaria una conversión a laparotomía en un caso, debido a que no se logró un pneumoperitoneo adecuado, por lo que no se obtuvo una buena visión. En este caso se realizó una anexectomía izquierda, debido a la presencia de un infarto anexial asociado a torsión.

Respecto a complicaciones del postoperatorio, sólo un caso presentó fiebre las primeras 48 horas, controlada con mediadas médicas. El alta hospitalaria fue en $6(67 \%)$ mujeres a las 48 horas de la cirugía. En 2 casos fue a las 72 horas y en 1 a las 96 horas. Esta última paciente presentó un cuadro febril, de resolución con medidas médicas a las 48 horas, descartándose factores infecciosos u otra complicación. Correspondía a un caso de triple torsión ovárica resuelta por destorsión y observación (Tabla III). Todos los embarazos evolucionaron normalmente poscirugía. No se reportan partos prematuros o pérdida reproductiva. El estudio biópsico diferido, realizado en 3 pacientes, demostró un quiste seroso paratubario, un quiste luteínico parcialmente hemorrágico y un infarto anexial.

Tabla I

CARACTERÍSTICAS DE LAS PACIENTES EN ESTUDIO

\begin{tabular}{cccccc}
\hline Caso & Edad & $\begin{array}{c}\text { Edad } \\
\text { gestacional }\end{array}$ & $\begin{array}{c}\text { Tipo de } \\
\text { embarazo }\end{array}$ & Embarazo & $\begin{array}{c}\text { Motivo de } \\
\text { ingreso }\end{array}$ \\
\hline 1 & 23 & 23 & espontáneo & DAA \\
2 & 36 & 19 & espontáneo & único & QP \\
3 & 31 & $5+5$ & espontáneo & gemelar & DAA \\
4 & 32 & $8+3$ & FIV & único & DAA \\
5 & 32 & 9 & FIV & único & DAA \\
6 & 30 & $5+5$ & IIU & único & DAA \\
7 & 31 & 27 & espontáneo & único & DAA \\
8 & 35 & 4 & IIU & triple & DAA \\
9 & 29 & 12 & IIU & DAA \\
\hline
\end{tabular}

FIV: Fertilización in vitro, IIU: Inseminación intrauterina, DAA: Dolor abdominal agudo, QP: Quiste persistente. 
Tabla II

DIAGNÓSTICO PREOPERATORIO Y RESOLUCIÓN

\begin{tabular}{cll}
\hline Caso & Diagnóstico preoperatorio & Cirugía \\
\hline 1 & Quiste anexial izquierdo: torsión & Quistectomía \\
2 & Quiste anexial derecho persistente & Ooferectomía \\
3 & Quiste anexial izquierdo: torsión & Destorsión ovárica \\
4 & Abdomen agudo & Punción, destorsión, fijación \\
5 & Abdomen agudo & Destorsión ovárica \\
6 & Quiste ovárico derecho: torsión & Destorsión ovárica \\
7 & Quiste ovárico izquierdo: torsión-infarto & Anexectomía \\
8 & Quiste ovárico izquierdo: torsión & Destorsión ovárica \\
9 & Quiste ovárico izquierdo: torsión & Destorsión ovárica \\
\hline
\end{tabular}

Tabla III

LAPAROSCOPIA Y ALTA HOSPITALARIA

\begin{tabular}{ccccc}
\hline Entrada & Conversión & $\begin{array}{c}\text { Tiempo } \\
\text { operatorio } \\
(\mathrm{min})\end{array}$ & Complicaciones & $\begin{array}{c}\text { Alta } \\
\text { (horas) }\end{array}$ \\
\hline Supraumbilical & No & 50 & No & 48 \\
Umbilical & No & 60 & No & 48 \\
Umbilical & No & 45 & No & 72 \\
Umbilical & No & 45 & No & 48 \\
Umbilical & No & 45 & No & 48 \\
Umbilical & No & 35 & Fiebre & 96 \\
Umbilical & Sí & 60 & No & 72 \\
Umbilical & No & 35 & No & 48 \\
Umbilical & No & 25 & No & 48 \\
\hline
\end{tabular}

\section{DISCUSIÓN}

La incidencia de quistes anexiales en un embarazo espontáneo, se estima en un $4 \%$, superior a la de 1 en 2.300 descrita por trabajos clásicos. Estas lesiones anexiales se asocian a algunas complicaciones como dolor, torsión y eventual cirugía, en un rango de 12 a $28 \%(11,12,14,21-25)$. Si a ello le agregamos el incremento actual en técnicas de fertilización asistida, con estimulación ovárica controlada, más el síndrome de hiperestimulación, la incidencia de lesiones anexiales y complicaciones relacionadas aumenta aún más (4,6-10). Aproximadamente un $3 \%$ de las emergencias ginecológicas corresponden a torsión anexial, y el 70 a $80 \%$ de ellas ocurre en edad reproductiva $(26,27)$.

En esta serie, más de la mitad de los casos corresponden a pacientes que fueron sometidas a terapias de fertilidad, con inducción de ovulación de distinta complejidad. Lo anterior, asociado a embarazo e hiperestimulación ovárica, incrementa los riesgos de torsión anexial, según diversos autores. Ovarios aumentados de volumen, quísticos, producto de inducción de ovulación, a los cuales se agrega el síndrome de hiperestimulación ovárica, tienen mayor riesgo de torsión $(4,8,10)$. El $\mathrm{SHO}$ complica el diagnóstico inicial, ya que estas pacientes se presentan con un abdomen distendido y sensible, secundario a ovarios aumentados de volumen. Al lograr embarazo, en el contexto de es- 
timulación ovárica y $\mathrm{SHO}$, los quistes persisten y se mantiene el riesgo de torsión $(9,10)$.

En nuestra casuística, todas las pacientes ingresadas por urgencia consultaron por dolor abdominal agudo, interpretado inicialmente como cólico nefrítico o probable torsión anexial. Lo interesante es que con esas presunciones, el estudio de laboratorio, en que destaca la ecotomografía abdominopelviana y transvaginal, rápidamente orientaron a la etiología anexial. Las imágenes quísticas, mixtas, de cuerpos lúteos o compatibles con hiperestimulación ovárica, más la clínica, fueron definiendo la conducta quirúrgica. La utilidad de la ecotomografía pelviana con o sin doppler, está descrita en la literatura. Es importante mencionar que la presencia de flujos no descarta la torsión anexial y que el doppler puede fallar en el diagnóstico hasta en un $60 \%$ $(28,29)$. En nuestra serie, el doppler no fue el elemento central en la evaluación de laboratorio, posiblemente debido a lo expresado anteriormente.

Con el diagnóstico definido, tratándose de una lesión anexial complicada en paciente embarazada, el punto a decidir fue la vía de abordaje. Esta revisión es clara en cuanto a que siempre se optó por la vía laparoscópica como técnica quirúrgica resolutiva. La seguridad y eficacia de la laparoscopia durante el embarazo ha sido estudiada in extenso (21-23). Al evaluar las distintas series podemos concluir que los beneficios superan a los riesgos. La principal ventaja de la laparoscopia es que se trata de una cirugía "cerrada", sin incisión abdominal, con tiempos operatorios reducidos, deambulación precoz y disminución de potenciales hernias incisionales, ente otros. Los riesgos se asocian a la eventual punción del útero, víscera hueca o grandes vasos. Aunque son escasas las publicaciones que informan estas complicaciones, siempre debemos tenerlas presentes $(30,31)$. Se trata de un riesgo que es mayor durante la entrada primaria, el cual puede reducirse al utilizar visión directa con cámara $(32,33)$. Respecto al CO2 y su absorción fetal, ha sido estudiado y no se considera un factor de riesgo en la actualidad (34-37). Las presiones intra-abdominales de $\mathrm{CO} 2$ se manejaron en un rango de 15 a $20 \mathrm{mmHg}$. En general, se recomienda no superar los $15 \mathrm{mmHg}$ en embarazos. En esta casuística, las mayores presiones se emplearon en las gestaciones más pequeñas y en los primeros casos. Lo anterior puede ser atribuible a una menor experiencia en este procedimiento o curva de aprendizaje.

Las edades gestacionales de estas pacientes oscilaron entre las 4 y 27 semanas. Los casos de edad gestacional menor, se asociaron a hiperestimulación ovárica, mientras que a mayores edades nos enfrentamos a lesiones anexiales persistentes.

Al analizar la técnica quirúrgica empleada, observamos que en la mayoría de los casos se procedió a la destorsión anexial, aún con anexos cuya observación planteó duda respecto a su indemnidad. Se reporta que en un $90 \%$ de los casos con anexos de aspecto necrótico o hemorrágico, la destorsión es efectiva, con recuperación de función ovárica. La fisiopatología de este evento es interesante. Primero se ve comprometido el retorno venoso, luego se produce una oclusión arterial parcial, hasta llegar a una total. Todo depende del grado de compromiso de la irrigación vascular principal y de la circulación colateral, lo que determinará la cuantía del daño isquémico ovárico. Se ha observado, del punto de vista funcional, después de una destorsión, que estos ovarios muestran desarrollo folicular y respuesta adecuada frente a su estimulación farmacológica, con fertilización oocitaria en procedimientos posteriores de fertilización in vitro. En concordancia con lo expuesto, diversas revisiones demuestran que a pesar de existir un retraso en el diagnóstico y tratamiento, las tasas de preservación de la función ovárica pueden ser altas (38). Otro elemento a favor de la destorsión ovárica es que en la literatura médica no hay informes de un incremento en el riesgo de eventos tromboembólicos.

En uno de nuestros casos se realizó punción de los quistes más destorsión. Al coexistir el SHO con la torsión, el puncionar los quistes tiene un doble efecto; facilita el procedimiento e impide una torsión posterior, al reducir el volumen de las masas anexiales. Además, contribuye al tratamiento del $\mathrm{SHO}$.

Conversión a laparotomía debimos realizar en solo un caso, que correspondía a un embarazo de 27 semanas con una torsión ovárica izquierda asociada a infarto anexial. En este caso, se utilizó la técnica de entrada con visión directa $(32,33)$. No se logró un neumoperitoneo adecuado ni una visión óptima. En la cirugía abierta se realizó una anexectomía izquierda, al encontrar un anexo totalmente infartado.

El límite seguro para cirugía laparoscópica y embarazo, según diversos autores, fluctúa entre las 26 y las 28 semanas, con casos exitosos hasta las 32 semanas (25). La limitación está dada por una visión inadecuada producto del útero grávido.

Los tiempos operatorios en esta serie, creemos que son razonables, con un rango de 25 a 60 minutos. Este elemento también contribuye a disminuir la morbilidad en estas pacientes.

En nuestra serie, el tiempo de hospitalización fue abreviado, con alta promedio a las 48 horas. 
Otras publicaciones recomiendan 24 horas de observación después de una destorsión laparoscópica. Algunas publicaciones proponen una ooforopexia para evitar torsiones recurrentes (39).

El no reportar complicaciones inmediatas 0 tardías, salvo un caso de fiebre moderada las primeras 48 horas, manejada clínicamente, nos hace plantear dos posibilidades: casuística reducida más una técnica quirúrgica adecuada. Aunque, también debemos mencionar que las potenciales complicaciones descritas por otros autores en este tipo de cirugía laparoscópica, no se ve incrementada por la condición de embarazo asociado, si se respetan los cuidados básicos de toda cirugía endoscópica $(24,40,41)$ y se tienen en cuenta las condiciones especiales dadas por el embarazo mismo.

En los casos en que se realizó alguna extracción operatoria y se realizó estudio de biopsia diferida, los resultados corresponden a lo reportado clásicamente, salvo que no tenemos quistes dermoides, que son frecuentemente mencionados en la literatura $(14,15,25)$. Tampoco hubo neoplasias malignas en nuestra serie.

Todos los embarazos evolucionaron fisiológicamente, lo que refuerza los beneficios de la técnica laparoscópica como enfrentamiento a esta entidad clínica.

En resumen, presentamos una cohorte de pacientes embarazadas portadoras de quistes anexiales, predominantemente complicados, que fueron manejados laparoscópicamente en forma exitosa. Destacamos la destorsión anexial como una técnica conservadora. El enfrentamiento debe realizarse con un criterio de conservación reproductiva, teniendo en cuenta la gran capacidad de los ovarios para resistir la injuria isquémica. Finalmente, es importante recordar que al asociarse el SHO y embarazo, se incrementa el riesgo de complicaciones anexiales, como la torsión, que debemos sospechar.

\section{CONCLUSIÓN}

Al igual como lo confirma la literatura científica internacional, a través de nuestra experiencia, podemos decir que la laparoscopia representa una opción válida cuando se requiere abordaje quirúrgico por un quiste anexial complicado o persistente, durante el embarazo. La torsión ovárica es la complicación más frecuente en pacientes embarazadas portadoras de quistes anexiales sintomáticos, que ingresan por urgencia. La destorsión simple, es una buena opción en el manejo de estas pacientes, si las condiciones lo permiten. Los quistes anexiales complicados, en esta serie, se asociaron frecuente- mente a embarazos producto de técnicas de fertilización asistida.

\section{BIBLIOGRAFÍA}

1. Stany M, Elkas J. Laparoscopy for adnexal masses in pregnancy? Cont Ob/Gyn 2007; 52(12): 44-9.

2. Hasiakos D, Papakonstantinou K, Kontoravdis A, Gogas $L$, Aravantinos L, Vitoratos N. Adnexal torsion during pregnancy: report of four cases and review of the literature. J Obstet Gynaecol Res 2008;34:683-7.

3. Kumari I, Kaur S, Mohan H, Huria A. Adnexal masses in pregnancy: a 5-year review. Aust N Z J Obstet Gynaecol 2006;46(1):52-4

4. Madill JJ, Mullen NB, Harrison BP. Ovarian hyperstimulation syndrome: a potentially fatal complication of early pregnancy. J Emerg Med 2008;35(3):283-6.

5. Rackow BW, Patrizio P. Successful pregnancy complicated by early and late adnexal torsion after in vitro fertilization. Fertil Steril 2007;87(3):697.e9-12.

6. Källén B. Maternal morbidity and mortality in in-vitro fertilization. Best Pract Res Clin Obstet Gynaecol 2008;22(3):549-58.

7. Källén B, Finnström O, Nygren KG, Otterblad Olausson $P$, Wennerholm UB. In vitro fertilization in Sweden: obstetric characteristics, maternal morbidity and mortality. BJOG 2005;112(11):1529-35.

8. Avecillas JF, Falcone T, Arroliga AC. Ovarian hyperstimulation syndrome. Crit Care Clin 2004;20(4):67995.

9. Eftekhar Z, Rahimi-Moghaddam P, Yarandi F, Tahmasbi M. An ovarian torsion in severe spontaneous ovarian hyperstimulation syndrome associated with a singleton pregnancy. J Obstet Gynaecol 2005;25(4):393-4.

10. Goshen R, Lavy Y, Hochner-Celnikier H, Milwidsky A. Third trimester torsion of persistent ovarian cyst following ovarian hyperstimulation--an unusual cause of preterm labor. Gynecol Obstet Invest 1992;33(4):2445.

11. Schmeler KM, Mayo-Smith WW, Peipert JF, Weitzen $S$, Manuel MD, Gordinier ME. Adnexal masses in pregnancy: surgery compared with observation. Obstet Gynecol 2005;105:1098-1103.

12. El-Yahia AR, Rahman J, Rahman MS, al-Suleiman SA. Ovarian tumours in pregnancy. Aust N Z J Obstet Gynaecol 1991;31(4):327-30.

13. Nowak M, Szpakowski M, Wilczyński JR. Ovarian tumors in pregnancy--proposals of diagnosis and treatment. Ginekol Pol 2004;75(3):242-9.

14. Chittacharoen A, Wangpusayavisut A, O-Prasertsawat $P$. Adnexal masses in pregnancy. J Med Assoc Thai 2005;88 Suppl 2:S37-40.

15. Türkçüoğlu I, Meydanli MM, Engin-Ustün $Y$, Ustün $Y$, Kafkasli A. Evaluation of histopathological features and pregnancy outcomes of pregnancy associated adnexal masses. J Obstet Gynaecol 2009;29(2):1079.

16. Yen CF, Lin SL, Murk W, Wang CJ, Lee CL, Soong YK, Arici A. Risk analysis of torsion and malignancy for adnexal masses during pregnancy. Fertil Steril 2009;91(5):1895-1902. 
17. Leiserowitz GS, Xing G, Cress R, Brahmbhatt B, Dalrymple JL, Smith LH. Adnexal masses in pregnancy: how often are they malignant? Gynecol Oncol 2006;101(2):315-21.

18. Giuntoli RL 2nd, Vang RS, Bristow RE. Evaluation and management of adnexal masses during pregnancy. Clin Obstet Gynecol 2006;49(3):492-505.

19. Hermans $\mathrm{RH}$, Fischer DC, van der Putten HW, van de Putte G, Einzmann T, Vos MC, Kieback DG. Adnexal masses in pregnancy. Onkologie 2003;26(2):167-72.

20. Jackson H, Granger S, Price R, Rollins M, Earle D, Richardson W, Fanelli R. Diagnosis and laparoscopic treatment of surgical diseases during pregnancy: an evidence-based review. Surg Endosc 2008;22(9):1917-27.

21. Azuar AS, Bouillet-Dejou L, Jardon $K$, Lenglet $Y, C a-$ nis $M$, Bolandard $F$, Storme B, Bonnin M, Pezet D, Buc E, Lemery D, Gallot D. Laparoscopy during pregnancy: experience of the French university hospital of Clermont-Ferrand. Gynecol Obstet Fertil 2009;37(78):598-603.

22. Al-Fozan H, Tulandi T. Safety and risks of laparoscopy in pregnancy. Curr Opin Obstet Gynecol 2002;14(4):375-9.

23. Palanivelu C, Rangarajan M, Senthilkumaran S, Parthasarathi R. Safety and efficacy of laparoscopic surgery in pregnancy: experience of a single institution. $J$ Laparoendosc Adv Surg Tech A 2007;17(2):186-90.

24. Mathevet $P$, Nessah K, Dargent D, Mellier G. Laparoscopic management of adnexal masses in pregnancy: a case series. Eur J Obstet Gynecol Reprod Biol 2003;108(2):217-22.

25. Morice P, Louis-Sylvestre C, Chapron C, Dubuisson JB. Laparoscopy for adnexal torsion in pregnant women. J Reprod Med 1997;42(7):435-9.

26. McWilliams GD, Hill MJ, Dietrich CS 3rd. Gynecologic emergencies. Surg Clin North Am 2008;88(2):265-83.

27. Promecene PA. Laparoscopy in gynecologic emergencies. Semin Laparosc Surg 2002;9(1):64-75.

28. Cilotti A, Weiss C, Bagnolesi P, Campassi C, Bimbi $\mathrm{M}$, Bartolozzi C. Echography in gynecologic emergencies. Radiol Med 1992;83(5):630-5.

29. Auslender R, Shen O, Kaufman Y, Goldberg Y, Bardi- cef M, Lissak A, Lavie O. Doppler and gray-scale sonographic classification of adnexal torsion. Ultrasound Obstet Gynecol 2009;34(2):208-11.

30. Rodrigues SP, van Hooff $\mathrm{MH}$, van Aggelen GP, de Vleeschouwer LH. Laparoscopy during pregnancy. Ned Tijdschr Geneeskd 2009;153(1-2):25-9.

31. Buser KB. Laparoscopic surgery in the pregnant patient: results and recommendations. JSLS 2009;13(1):32-5.

32. Ricci P, Solà V, Pardo J. Entrada umbilical con trocar mínimamente invasivo bajo visión directa en laparoscopia ginecológica. Rev Chil Obstet Ginecol 2008;73(2):104-9.

33. Troncoso JL, Ricci P, Susaeta R, Devoto JC. Torsión anexial y embarazo: Resolución laparoscópica mínimamente invasiva, con entrada bajo visión directa. Rev Chil Obstet Ginecol 2008;73(4):277-82.

34. Lenglet $Y$, Roman $H$, Rabishong $B$, Bourdel $N$, Bonnin M, Bolandard F, Duband P, Pouly JL, Mage G, Canis $M$. Laparoscopic management of ovarian cysts during pregnancy. Gynecol Obstet Fertil 2006;34(2):101-6.

35. O'Rourke N, Kodali BS. Laparoscopic surgery during pregnancy. Curr Opin Anaesthesiol 2006;19(3):2549.

36. Barnard JM, Chaffin D, Droste S, Tierney A, Phernetton $\mathrm{T}$. Fetal response to carbon dioxide pneumoperitoneum in the pregnant ewe. Obstet Gynecol 1995;85:669-74.

37. Curet MJ, Vogt DA, Schob O, Qualls C, Izquierdo LA, Zucker KA. Effects of CO2 pneumoperitoneum in pregnant ewes. J Surg Res 1996;63(1):339-44.

38. White M, Stella J. Ovarian torsion: 10-year perspective. Emerg Med Australas 2005;17(3):231-7.

39. Djavadian D, Braendle W, Jaenicke F. Laparoscopic oophoropexy for the treatment of recurrent torsion of the adnexa in pregnancy: case report and review. Fertil Steril 2004;82(4):933-6.

40. Fatum M, Rojansky N. Laparoscopic surgery during pregnancy. Obstet Gynecol Surv 2001;56(1):50-9.

41. Rojansky N, Shushan A, Fatum M. Laparoscopy versus laparotomy in pregnancy: a comparative study. J Am Assoc Gynecol Laparosc 2002;9(1):108-10. 